\title{
Drawing a Panoramic Imaginary
}

DANIELE PROFETA

Syracuse University
Throughout history forms of representation have profoundly shaped the way we see and operate within the built environment. Today, Google Street View (GSV) can be understood as one of the most ubiquitous modes of orientation in our daily life, radically transforming the way in which we engage with the world around us. With this paper I will position GSV within a long lineage of immersive media, from panoramas to early cinematic images, to move past ideas of 'objective representation' and to highlight its potential as a site for design investigations. In order to foreground the relevance of these contemporary imaging technologies and immersive platforms of vision as instigators of a disciplinary conversation on representation, I will present some of my students' work alongside 'Arctic LiDAR', an immersive video installation I am currently developing. Here the tension between the aesthetic realms of human and machinic vision, the collapse of multiple territories in a composite digital model and the temporary displacement of viewers to remote and inaccessible sites, much like the infrastructure provided by GSV, become critical tools to shape contemporary subjectivities. Immersive representation to move from the known to the unknown.

What does it mean that this seamless panning and zooming [in a geo-browser like Google Earth] has become naturalized, that it has become how I see and experience the world, or how I want to see and experience my world?

-Todd Presner, HyperCities: Thick Mapping in the Digital Humanities

\section{INTRODUCTION}

While zooming-in and zooming-out in a multi-perspectival digital bubble seems to have become the ubiquitous mode of self-orientation, this paper exploits the notion that such environment, far from being a purely descriptive, passive representation of an 'external reality', it performs as a cognitive agent of cultural organization. A strange loop is in place here: our world-views afford the development of specific modes of representation, of engagement with the world, and in turn they begin to have an impact in that same world, becoming an active element in the way we understand it. Put more simply, it is the technologies through which we see and experience the world that define the way we construct it. With this paper, I want to investigate the role that immersive digital environments, inhabited through networked media, ubiquitous feeds of information and ever more pervasive modes of vision, have in shaping our public identity and how they should be considered crucial sites for design explorations. More specifically, I will take Google Street View (GSV) as a contemporary example of an immersive system of representation, one that engaging with a long lineage of visual strategies is radically transforming our sensory experience of the world. With a critical understanding of these modes of representation, we can begin to suggest ways to intervene within them, exposing existing realities and speculating on the possible futures of our built environment. Panoramas, and their contemporary, digitally constructed equirectangular views, will be investigated as devices that allow us to draw things together: multi-faceted, composite images "to make a difference at all in the way we argue, prove and believe."

\section{IMMERSIVE MEDIA AND PUBLIC IDENTITIES}

To better understand the representation strategies at play in a platform such as Google Street View, it is very helpful to first consider some of the precedents that contributed in constructing what we might define as the imaginary of the panoramic, one that leverages illusionistic strategies to temporarily displace its viewers, to immerse them in an environment as a storytelling media.

It is important to point out here that while I will focus on the historical case of the Panorama as it appeared in London, there have been many other examples of immersive imaging strategies. From the linear distribution of images and texts (hieroglyphics) of the ancient Egyptian Book of the Dead (around $1550 \mathrm{BCE}$ ) to the sequential art carved out across the Trajan's Column as narration of the Dacian Wars (113 CE) or the Japanese hand scrolls of the 17th century deploying environments, events and characters to produce immersive narrative experiences, this is a global conversation that goes beyond the limited narrative of the Western Hemisphere, but one that would exceed the scope of this paper.

One of the first appearances of the Panorama as a viewing experience can be found in a temporary building in Leicester Square in London (1791). The work of Robert Baker, a painter that patented in 1787 an "apparatus, which I call La Nature à Coup d'Oeil,"2 was received with incredible public interest. The magical feeling of temporary displacement to a scene painted with a high level of realism, structured around a multi-perspectival composition across the stretched canvas, bringing you 'as if really there', left the visitors incredulous. Much has been written about the 


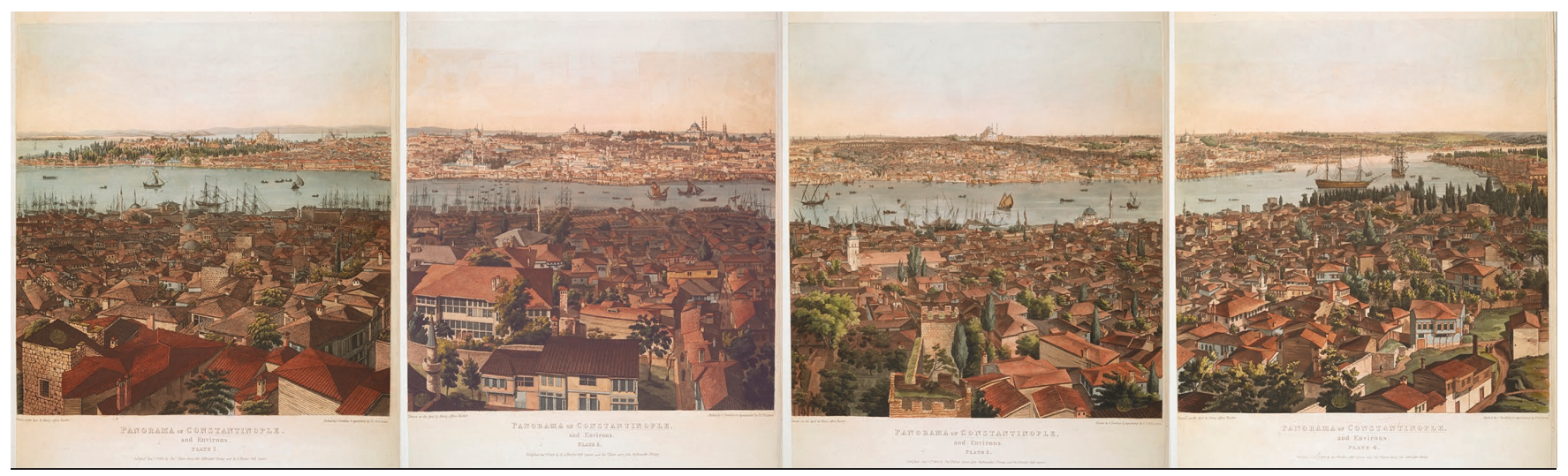

Figure 1. Plates 1 to 4 from Panorama of Constantinople, and environs. Henry Aston Barker, Charles Tomkins, etching and aquatint. London: Thos. Palser, Surry Side Westminster Bridge (1813). Library of Congress Control Number 2012648911.

history, ${ }^{3}$ cultural practices ${ }^{4}$ and media archeologies ${ }^{5}$ of this immersive device, tracing its introduction as a new art form that quickly began to engage with mass culture and a market for mediated realities. Drawing from this existing body of research, I would like to focus on a series of spatial and representational features, together with their associated modes of engagement, that will help us both to understand some of the strategies at play within Google Street View, as well as to identify a few productive sites of speculation: opportunities "to think again or anew [...] to re-enchant our (representational) tools."

Let's take as an example Barker's first permanent panorama from 1793, the Panorama Building. Housed within a cylindrical enclosure, visitors would climb up within an enclosed stairway into a viewing platform at the center of the building, where they would find themselves surrounded by a colossal painting of a featured location stretched along the inner walls of the volume. By hiding the margins of the canvas, controlling diffuse lighting pouring from above and with a careful attention towards the enhanced realism of the presented image, the viewers were immersed in a "three dimensional virtual reality that extends in complete circle around [them], to an imagined horizon and then to an implied beyond." Particularly relevant here is the newly acquired position of the viewer to the offered landscape: elevated above a floating platform, simultaneously detached and in control of the surrounding scenery they acquired mastery over that world. Huhtamo brings our attention to the simultaneous appearance of Jeremy Bentham's idea of the Panopticon, highlighting the parallel between the figure of the guard and the emerging urban spectator. ${ }^{8}$ If, as Miller suggests, panoramas engaged with the nineteenth-century growing desire for visual control over a rapidly expanding world, ${ }^{9}$ it is crucial to underline that such vision in turn helped shaping ideological and commercial concerns that continue to inform many contemporary forms of immersive representation. The visitors' ambulatory movement transported them to distant locations, often both in space and time, beginning to construct what could be understood as global subjectivities which induced as much a sense of disorientation and confusion as of mastery and control.

It is also worth noticing that, while being transported to a series of exotic locations, such as distant geographical sites or historically relevant battlegrounds, the composite multi-perspectival images that the visitors engaged with flattened many of the hierarchies often found in urban representations. Here landmarks were juxtaposed to everyday elements of the city, so much so that chimneys, religious sites and property fences were depicted with the same obsession towards detailed realism. Collecting a remarkable number of elements from the chosen site, these images captured a visual record of the everyday urban environment further enhancing the illusion of temporary displacement: orbiting around the seemingly infinite canvas of these paintings the visitors would go through a continuous act of discovery, each infusing individual narratives upon the densely mapped environment.

Such ambition for an active immersion, over the following centuries, will take several forms, from the pictorial to the photographic space; ${ }^{10}$ among them, one of the most relevant examples for our investigation of the panoramic imaginary deployed by Google Street View and its role in reconfiguring the relationship between people, the built environment and its representation can be found in the early cinematic experiments with the moving image. Catalogued under the larger title of 'Panoramas' and 'Panoramic Views', between 1896 and 1912 we witness a remarkable production of cinematic practices aimed to portray immersive environments through very similar visual strategies as observed in their traditional painted antecedents. Huhtamo highlights this lineage with the direct relationship between circular panoramas and the successive examples of moving panoramas as the establishment of a "history of screen practices"11 leading towards these early cinematic panoramic views. In 


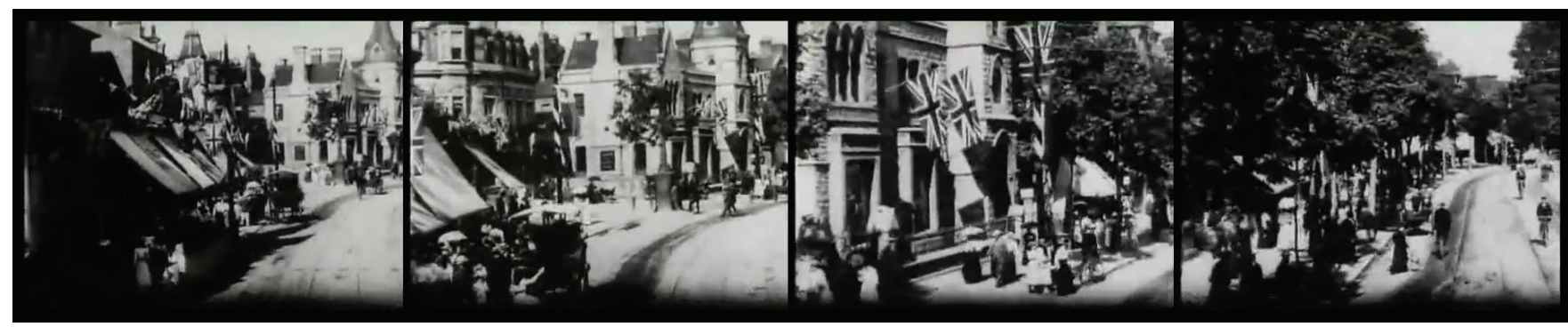

Figure 2. Stills from Panorama of Ealing from a Moving Tram. Film. Directed by William K.L. Dickson. London: British Mutoscope \& Biograph Company, 1901.

the moving panorama apparatus, Baker's surrounding environment was replaced with a single frame in which a long roll of a painting was mechanically moved across to present to the spectators, now sitting in an auditorium, a window into another world. In a similar manner, the filmed panoramas were constructed through a series of steady tracking shots where the camera, mounted on slow moving trains or horse drawn wagons, continuously rotated laterally to capture an immersive view of the street. In either scenarios, the rotating canvas or camera, suggests a shift from the movement of the spectator to the mobilization of the image itself as a mode to gain access to a particular space. As Abend underlines, in the case of the cinematic panoramas, the rotation of the camera also plays an important role in developing a different spatial engagement with the scene portrayed: ${ }^{12}$ the focus of attention of the viewers is shifted from the vanishing point implied by the linear movement of the transportation device along the city set and it stimulates instead a lateral exploration of the scene. The ritual of traveling, of active visual discovery of the urban environment remains here central, it transcends the fixed directionality of the scene and embodies what Friedberg identifies as the emergent mobile spectatorship of the 20th century flâneur. ${ }^{13}$

It is also particularly interesting to note how some of these first examples of cinematic panoramas, especially the 1904 series of several street-panoramas sponsored by the American Mutoscope and produced by the Biograph Company, have been understood as visual mapping practices of urban environments. ${ }^{14}$ If Benjamin argues that the visual space of the Panorama was once devised for transporting its visitors towards the countryside, ${ }^{15}$ where the city dwellers could demonstrate their supremacy over the territory of the provinces, the cinematic panoramas were deployed here as a systematic urban mapping project. A camera mounted upon a wagon, effectively surveying one by one a series of streets of downtown St. Joseph, Missouri, so that remote viewers could temporarily immerse themselves and once again navigate the urban traffic. This is a far too familiar system of representation, and it is crucial to recognize this lineage to understand how ever-more pervasive, visually engaging contemporary media platforms continually shape our immersive engagement to the represented world.

\section{ON GOOGLE STREET VIEW (AND ITS EXCEPTIONS)}

Google Street View can be understood as one of the most ubiquitous contemporary modes to navigate our built environment: immersed in a multi-perspectival digital bubble, we orient ourselves through a series of Geo-tagged, algorithmically edited, composited images, temporarily displacing ourselves to a familiar place as a way to organize our daily life, or to a yet unexplored landscape we are planning to visit. Through the interface of our browser or mobile device we begin to inhabit an environment that, in as much as it claims to objectively portray "your world - like you've never seen it,"16 it is actively transforming our understanding as well as our relationship with it.

Launched in 2007, Google Street View (GSV) is an online platform available as part of the giant Google Maps infrastructure, deployed to provide street-level coverage, allowing its users to virtually jump into a $360^{\circ}$ degree, fully maneuverable panoramic image. GSV imagery is acquired by omnidirectional cameras mounted on a wide variety of vehicles, ranging from the initial Street View Car to the more recent Street View Trekker, Trolley, Snowmobile and Trike, ${ }^{17}$ covering an evergrowing territory of the built environment. If the shift from a static painted panorama to the moving picture of its cinematic examples translated from an ambulatory engagement of the platform space to a lateral visuality as mode of exploration, here we enter a navigable space through a dynamic Graphic User Interface, bringing together the device of the map with the immersive imagery of GSV. Orbiting freely from a suspended point of view (the way cameras are mounted above their transportation device often results in an unusually elevated perspective) and provided with overlaid arrows to seamlessly navigate across this thick spatial representation, the user is confronted with what Gigle defines as "image as experience, ${ }^{18}$ one that prefigures embodied perception.

A significant body of research has already been developed around GSV as an extension of Geographic Information Systems (GIS), ${ }^{19}$ leveraging its systematic way to survey the territory as well as its aspiration towards a synchronic mapping of everyday spaces, a real-time dataset that could merge into CCTV practices. ${ }^{20}$ This type of studies follow the same trajectory of many investigations which leveraged virtual visualizations of geotagged imagery, such as Google Earth, 


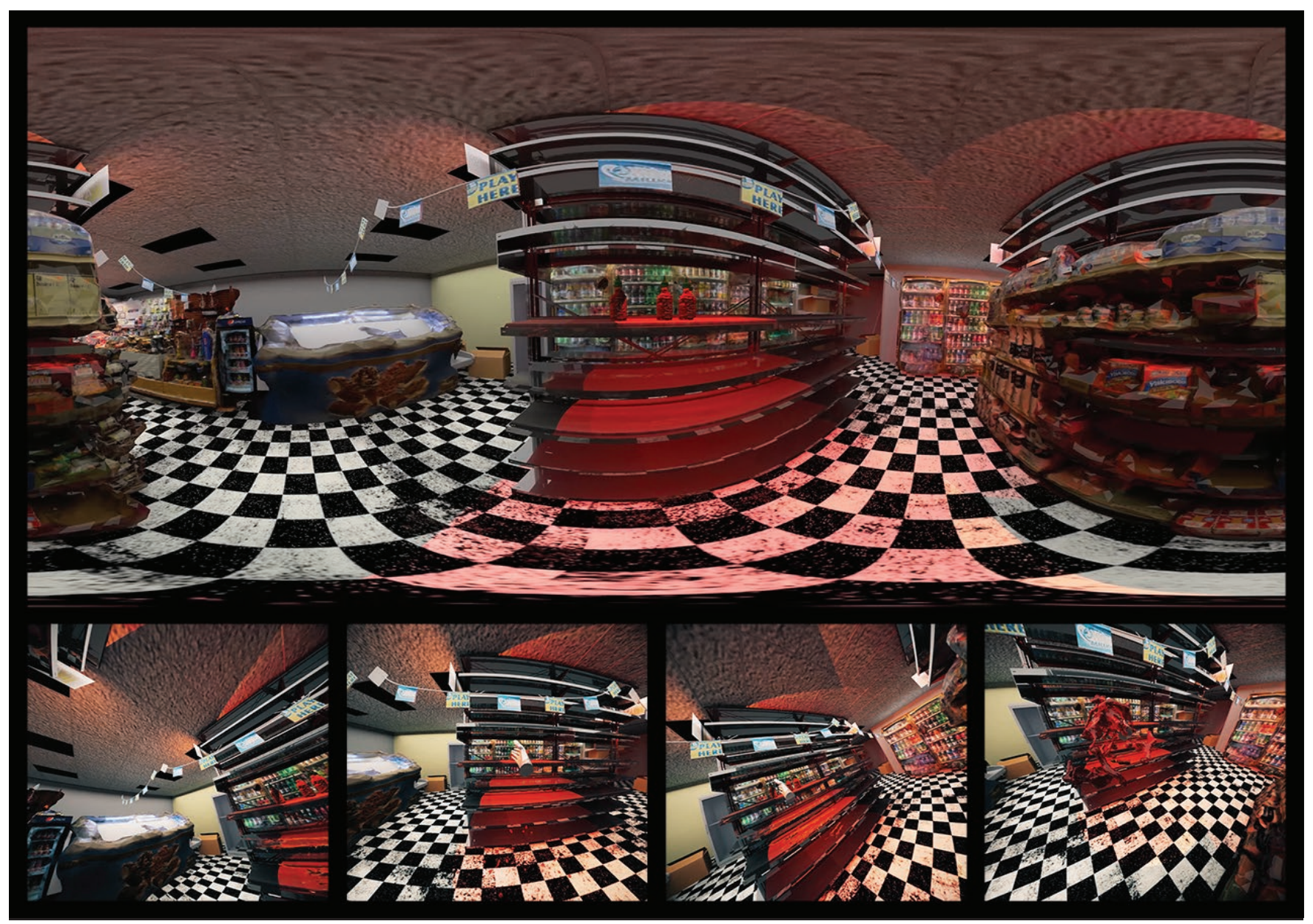

Figure 3. Stills from 'Shopping en la bodega' - 360 video by Geraldine Vargas and Pattaraporn Kittisapkajon (2017).

to engage with the ever-growing dataset of information collected on the territory. ${ }^{21}$ In order to move beyond tactics of mastery and control, it is crucial to recognize that the space of representation of GSV, conflating mapping and urban photography, acts as an agent of cultural organization, allowing for the creation of new realities rather than simply conceptualizing a pre-existing one. ${ }^{22}$ How can the immersive interface of GSV help to shape our relationship with the environment? And how can we leverage this mode of representation to speculate on the futures of our cities?

Pegman $^{23}$ is a useful character in the development of my argument. As a design problem Pegman, the little orange icon devised by Google to drop in the viewer in the panoramic site, embodies many of the opportunities that an immersive platform like GSV affords in shaping contemporary subjectivities. Its primary focus was to connect the users to their experience of discovery: clarity of its placement, so as to make sure the viewers would clearly understand where they would stand in the map, as well as its frontal orientation, to highlight their focus of vision while orbiting in the limitless digital canvas were some of the primary concerns in the development of this icon. An interview with Ryan Germick, ${ }^{24}$ the lead designer of the Doodle Team at Google, reveals how the 32 pixels available to represent the viewers' position and interaction could suggest ways to shape our relationship with this mediated world. The design iterations that finally brought us the orange figure ranged from a pair of Eyeballs, embodying a long tradition of modern visuality, to a Peg-woman, believe it or not - leveraging the lineaments of a stereotypical female body to indicate a clear frontal directionality. One of the most relevant here for our discussion is the abstracted figure of a humanoid whose head and torso are replaced by a photographic device: the icon of the visitors engaging with their surrounding world through a newly acquired machinic eye, one that automatically shots, stitches, blurs and geotags a series of punctual and yet immersive small-worlds. When we click-and-drag the Pegman, leaving it dangling in this spatial construct, can we project our position within these worlds beyond its absolute center? Can we recognize our agency as humans alongside the many others at play in the development of this space?

The construction of another point of view for this panoramic imaginary, one that attempts to displace anthropocentric views of the world, could be found in the instrumentalization of GSV by the Faroe Island Tourism Department as promoting device. 


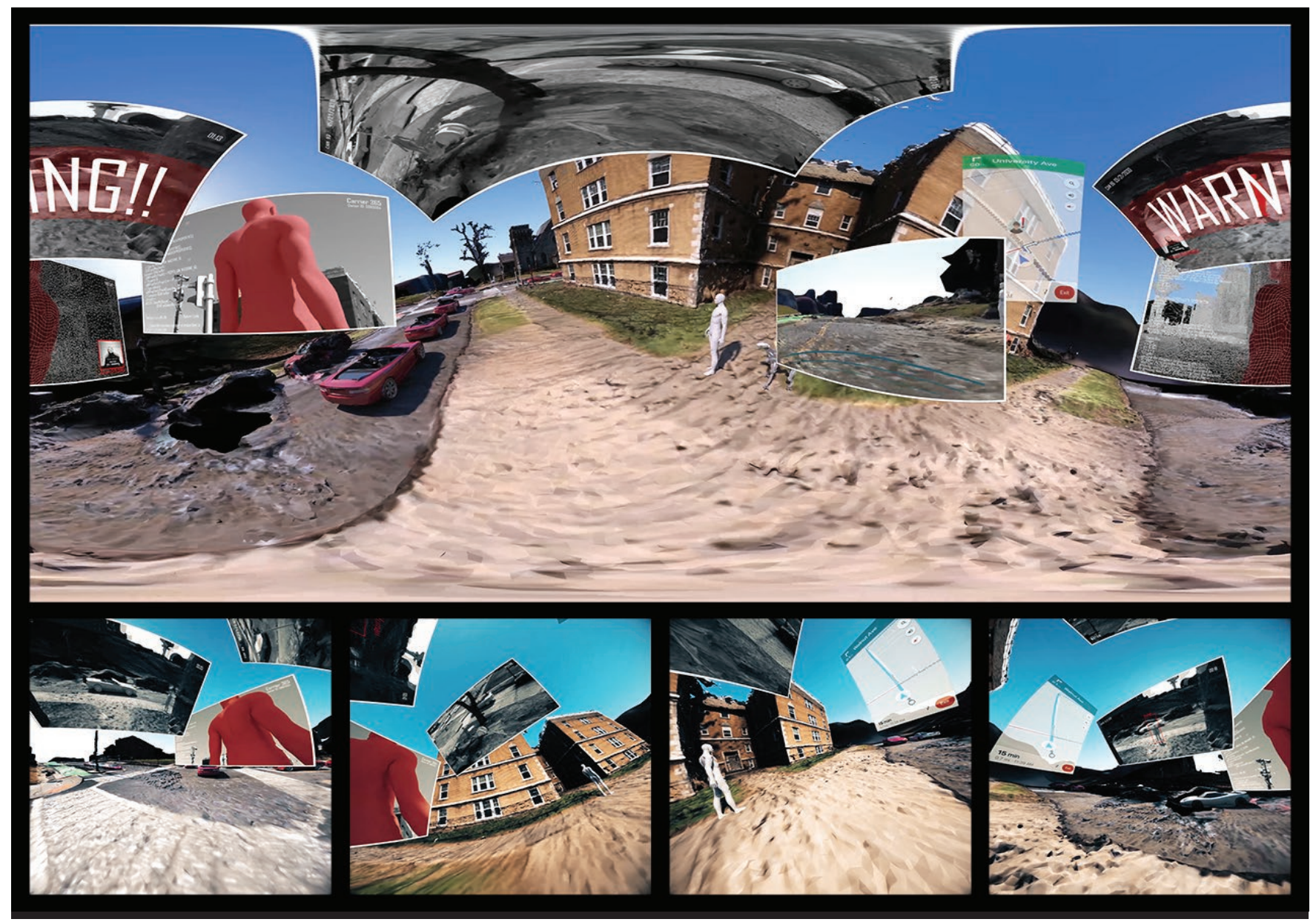

Figure 4. Stills from 'Machine Maniac' - 360 video by Miao Hui and Sibo Fei (2017).

Sheep-Street-View, ${ }^{25}$ a project named after its primary nonhuman actors, touches upon another significant issue behind this infrastructure of vision: predicated around a non-hierarchical organization of its content, GSV often reinforces many of the pre-existing inequalities of access to information. For example, to the date of this writing, the coverage of its panoramic platform remains primarily focused upon a western-centered world, leaving wide areas of inaccessibility: the Faroe Islands were one of them. To remediate this lack of accessibility, and leveraging the possibility of publishing user-generated content, a series of omnidirectional cameras were mounted above five wild sheep then released to freely browse the countryside. The suspended god-like point of view of the first aerial Panoramas of London is replaced with a close to the ground perspective of a tortuous landscape; the linear movement of the cinematic experiments and the regulated speed of the GSV fleet find their counterpart in the erratic exploratory motion of the sheep, suggesting new modes of engagement with the territory. Ultimately, while leaping into one of these $360^{\circ}$ sheep-led-environments, one is left wondering: what kind of worlds can we re-imagine from this newly acquired point of view? How can this immersive platform of vision open up new design and aesthetic opportunities in our contemporary landscape?

\section{SPECULATIONS ON GOOGLE STREET VIEW AND ARCTIC LIDAR}

If the lineage drawn this far suggests that the immersive mediated reality offered by GSV continuously shape the way we engage with the world around us, in the concluding passages of this paper I would like to present a few case studies where such panoramic imaginary has been leveraged as a representational tool: a venue of design to expose present realities as well as to speculate on possible futures.

First, I want to quickly talk about a few examples from the work developed in one of my research seminars titled 'Speculations on Google Street View' where the students were asked to collectively build small immersive worlds: leveraging contemporary surveying technologies, not unlike the ones deployed by GSV, they produced $360^{\circ}$ videos to suggest alternative world-views beyond a human-centric position of mastery and control. In 'Shopping en la Bodega', the viewers are displaced into an immersive digital shopping environment in which the agency of the cookie, collecting traces from our everyday digital-life, our geo-tagged Google searches and our double-taps upon preferred content, takes over our engagement with the products displayed. In the 


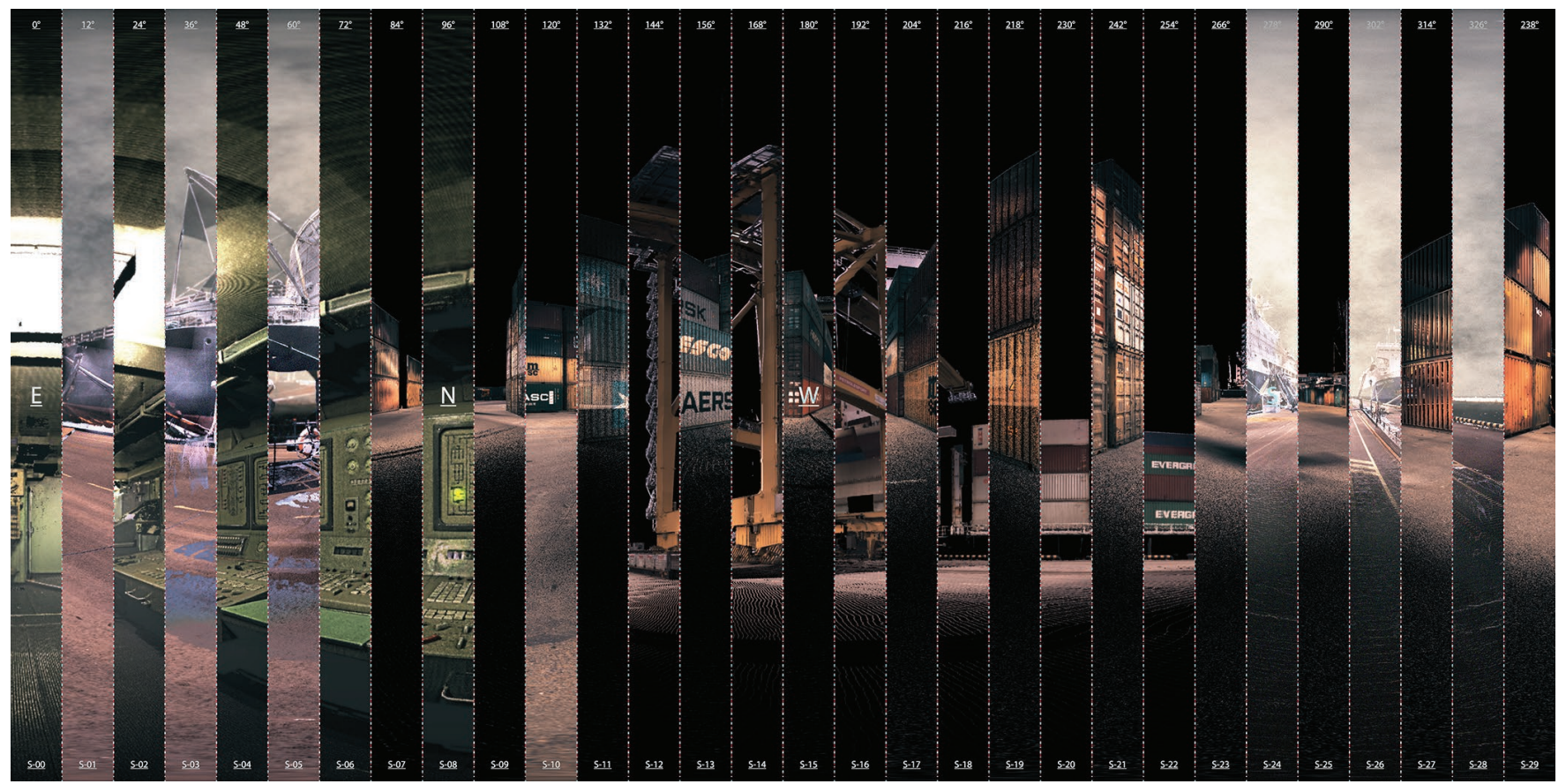

Figure 5. Composite Landscape from 'ARCTIC LiDAR' - 360 video by author (2018)

video, the fixed horizontal horizon characteristic of the panoramic antecedents, grounding the viewers into their elevated position of control, is only one of the many possible position of engagement with the world at hand: omniscient vantage points co-exist with partial, localized perspectives, requiring the viewers to constantly negotiate their position throughout the short movie. While navigating the Bodega, the viewers are confronted with a strangely mundane environment, in which the homogeneously distributed level of detail forces them to actively construct their narrative, to re-assemble some of the clues pointing towards a slight recalibration of their position as subjects of that world. In the progression of the scenes, the known tiling of the floor gets entangled with the QR codes of the products pre-selected by the cookie, in a motion that slowly projects them towards an unknown territory, one in which their actions are recognized as the result of a much larger ecology of human and not-human actors. The diffuse, controlled natural lighting is replaced with hue-saturated, focused highlight zones, forcefully leading our motion of visual exploration across the digital landscape.

In 'Machine Maniac' this landscape becomes even harder to navigate. Conflating the perspectives of CCTV feeds, GPS tracking databases, machinic and human vision, the viewers find themselves suspended outside of the represented subject. Throughout the short movie, the clear elevated view, similar to the one we might experience leaping in and out of Google Street View surveyed sites, is occluded by a rapidly expanding series of other perspectives. Reflecting on the ever-expanding media ecology that constructs our everyday life, the panoramic construct here is deployed to favor a sense of disorientation over control: visual hierarchy is flattened, and the once central point of view is multiplied beyond our embodied perception.

Finally, I would like to conclude by discussing the way in which panoramic vision becomes a storytelling device in 'ARCTIC LiDAR', a video installation that I am currently developing. Using $3 \mathrm{~d}$ scanning LiDAR to capture a series of remote locations along the Arctic Coast, the short movie becomes an immersive exploration of the quickly expanding logistic network of this region. Part documentary, part projective narrative, this $360^{\circ}$ video assembles multiple sites in a composite, speculative landscape that goes beyond direct presentation.

The machinic vision of the LiDAR scanner, capturing RGB values alongside a detailed $3 \mathrm{~d}$ map of the surveyed site, brings the viewers in the automated landscape of logistics, where driverless trucks and robotic cranes operate through the dense point cloud. Moving across the territory, this operative mode of vision is slowly contaminated by atmospheric elements foreign to the machinic eye. The viewers are caught in an ambiguous territory where a bodily experience of this harsh landscape and its extreme climate re-asserts their presence in an otherwise human-less environment. And yet, their point of view is constrained to the rigid linear movement of what appear to be indifferent machines, reinforcing the agency of the automated infrastructure upon the construction of this territory. 
What is at stake in these panoramic imaginaries is their capacity to suggest different contemporary subjectivities, together with different ways to engage with the environment around us. Immersive drawing devices to move from the known to the unknown.

\section{ENDNOTES}

1 Bruno Latour, "Visualisation and Cognition: Drawing Things Together" in Knowledge and Society Studies in the Sociology of Culture Past and Present, ed., H. Kuklick (Greenwich: Jai Press, 1986), 1-40.

2 "Apparatus for Exhibiting Pictures" British Patent no. 1612, granted June 19, 1787.

3 Charles Musser, "Toward a History of Screen Practice," Quarterly Review of Film Studies 9, no. 1 (Winter 1984).

4 Angela Miller, "The Panorama, the Cinema, and the Emergence of the Spectacular," Wide Angle 18, no. 2 (1996).

5 Erkki Huhtamo, Illusions in Motion: Media Archeology of the Moving Panorama and Related Spectacles (Cambridge, MA: The MIT Press, 2013).

6 James Bridle, A New Dark Age: Technology at the End of the Future (New York: Verso Books, 2018).

7 Peter Otto, "Between Virtual and the Actual: Robert Baker's Panorama of London and the Multiplication of the Real in the Late Eighteenth Century London," Romanticism on the Net 46 (May 2007).

8 Huhtamo, Illusions in Motion, 5.

9 Miller, "The Panorama, the Cinema, and the Emergence of the Spectacular," 34-69.

10 For more details see William Uricchio, “A 'Proper Point of View': The Panorama and Some of its Early Media Iterations," Early Popular Visual Culture 9, no. 3 (2011).

11 Musser, "Toward a History of Screen Practice," 59.

12 Pablo Abend, Geobrowsing: Google Earth und Co. - Nutzungspraktiken einer digitalen Erde (Bielefeld: Transcript Verlag, 2013), 141-143.

13 Anne Friedberg, Window Shopping. Cinema and the Postmodern (Berkley: University of California Press, 1993).

14 William Uricchio, "Panoramic Visions: Stasis, Movement, and the Redefinition of the Panorama," in La Nascita dei Generi Cinematografici / The Birth of Film Genres, eds., L. Quaresima, A. Raengo, and L. Vichi (Udine: Forum, 1999).

15 Walter Benjamin, "Paris, the Capital of the Nineteenth Century," in Benjamin, The Arcades Project, trans., Howard Eiland and Kevin McLaughlin (Cambridge, MA: Harvard University Press, 2003).

16 Google Maps “About” page, accessed September 19th, 2018. https://www. google.com/maps/about/.

17 Google Street View "Sources of Photography" page, accessed September 19th, 2018. https://www.google.com/streetview/understand/

18 Cheryl Gilge, "Google Street View and the Image as Experience," in GeoHumanities 2, no. 2 (2016).

19 Jacqueline W. Curtis et al., "Using Google Street View for Systematic Observation of the Built Environment: Analysis of Spatio-Temporal Instability of Imagery Dates," in International Journal of Health Geographics 12, no. 53 (2013).

20 Ben Campkin and Rebecca Ross, "Negotiating the City through Google Street View," in Camera Constructs: Photography, Architecture and the Modern City, eds., A. Higgott and T. Wray (Burlington: Ashgate, 2012), 147-57.

21 Steven J. Whitmeyer et al., eds., Google Earth and Virtual Visualizations in Geoscience Education and Research (Boulder: Geological Society of America, 2012)

22 George Lakoff and Mark Johnson, Metaphors We Live by (Chicago: University of Chicago Press, 1980).

23 Google, “Google Maps Street View - Launch Video," YouTube, 01:43, May 29, 2007. https://www.youtube.com/watch?v=91wuBqlny50.

24 Justine Sharrock, "Pegman: Google's Weird Art Project Hidden In Plain Sight," BuzzFeedNews, April 11, 2013. https://www.buzzfeednews.com/article/ justinesharrock/pegman-googles-weird-art-project-hidden-in-plain-sight.

25 "Sheep360," Visit Faroe Islands official website, accessed September 19th, 2018. https://visitfaroeislands.com/sheepview360/. 